\title{
Toll-Like Receptor 3 as a Recurrence Risk Factor and a Potential Molecular Therapeutic Target in Colorectal Cancer
}

This article was published in the following Dove Press journal: Clinical and Experimental Gastroenterology

Tatsuya Yoshida (D
Takuya Miura (D)
Tomoh Matsumiya ${ }^{2}$
Hidemi Yoshida ${ }^{2}$
Hajime Morohashi
Yoshiyuki Sakamoto'
Akira Kurose iD ${ }^{3}$
Tadaatsu Imaizumi
Kenichi Hakamada'
'Department of Gastroenterological
Surgery, Hirosaki University Graduate
School of Medicine, Hirosaki, Aomori
036-8562, Japan; ${ }^{2}$ Department of
Vascular Biology, Hirosaki University
Graduate School of Medicine, Hirosaki,
Aomori 036-8562, Japan; ${ }^{3}$ Department of
Anatomic Pathology, Hirosaki University
Graduate School of Medicine, Hirosaki,
Aomori 036-8562, Japan

Aomori 036-8562, Japan
Correspondence: Kenichi Hakamada Department of Gastroenterological Surgery, Hirosaki University Graduate School of Medicine, 5 Zaifu-cho, Hirosaki, Aomori 036-8562, Japan

Tel +8I I72 395079

Fax +8I 172395080

Email hakamada@hirosaki-u.ac.jp
Purpose: Colorectal cancer (CRC) often recurs after curative resection. Identification of major risk factors for CRC recurrence is important for effective prevention and treatment. In this study, we examined the potential relationship between CRC and TLR3 as this remains unclear.

Patients and Methods: Correlations between TLR3 immunostaining and clinicopathological factors and prognosis were examined in 50 samples that were randomly extracted from 264 patients with CRC from January 2010 to December 2011. Chemokines induced by TLR3 agonist stimulation were also examined using TLR3-positive human CRC cell lines. Furthermore, the association between TLR3 and chemokine expression was assessed by analyzing the immunohistochemistry of surgical specimens.

Results: Of the 50 patients, 14 (28\%) were TLR3-negative. In the comparison of clinicopathological factors between the TLR3-negative and -positive groups, there were more lymph node metastasis-positive cases in the TLR3-negative group, and this difference was significant. Furthermore, there was no difference in overall survival rates between the two groups, but the 5-year recurrence-free survival (RFS) was significantly lower in the TLR3negative group (46.2\%) than in the TLR3-positive group (78.1\%). Analysis of 5-year RFS using factors thought to be related to recurrence identified a high tumor budding and a TLR3negative status as independent risk factors for recurrence. TLR3 activation of CRC cell lines induced expression of C-C motif chemokine ligand 2 (CCL2), C-C motif chemokine ligand 5 (CCL5), and interleukin-8. The expressions of CCL2, CCL5, and IL-8 were observed in the TLR3-positive tumor cells of surgical specimens.

Conclusion: Non-expression of TLR3 in CRC cells was associated with lymph node metastasis and was an independent risk factor for recurrence. These results suggest that TLR3 may not only be used as a prognostic factor and a risk factor for recurrence, but further studies on the involvement of TLR3 with tumor growth may provide new therapeutic strategies.

Keywords: colorectal cancer, toll-like receptor 3, recurrence, CCL2, CCL5, IL-8

\section{Introduction}

Colorectal cancer (CRC) is a common malignant neoplasm with the third highest prevalence worldwide, after lung cancer and breast cancer. It has the second highest mortality rate after lung cancer. ${ }^{1}$ Hereditary CRC accounts for $10 \%$ of the cases, while all other cases are sporadic. ${ }^{2}$ Chronic inflammation and lifestyle and eating habits, including unchecked obesity, alcohol and red meat consumption, have been suggested as risk factors for $\mathrm{CRC} .^{3-6}$ 
CRC often recurs after curative resection, with recurrence rates reaching $13.3 \%$ in Stage II and $30.8 \%$ in Stage III cases. $^{7}$ Therefore, it is important to evaluate the recurrence risk in individual cases and introduce adjuvant chemotherapy in addition to curative resection in CRC treatment strategies. The numbers of dissected lymph nodes $(<12)$, T4, perforation cases, and poorly differentiated carcinoma in Stage II have recently been identified as high-risk factors for recurrence, ${ }^{8,9}$ but none of these factors is molecular in nature to serve as a therapeutic target. Therefore, there is the need to identify a molecule that is a strong recurrence risk factor to serve as a direct treatment target.

Toll-like receptors (TLRs) are pattern-recognition receptors, and 13 types have been found in mammals. ${ }^{10}$ TLRs recognize the pathogen-associated molecular patterns (PAMPs) of bacteria-derived lipopolysaccharides and virusderived DNA and RNA, and induce proinflammatory cytokines in mechanisms that are important for the activation of the innate immune system. ${ }^{11}$ Similar to exogenous pathogenderived PAMPs that induce inflammation, Matzinger proposed that endogenous molecules released from injured cells, termed damage-associated molecular patterns (DAMPs), can also induce inflammation. ${ }^{12}$ Many DAMPs have now been identified, including high mobility group 1 (HMGB1) and uric acid. ${ }^{13-15}$ Sato et al suggested that in the tumor microenvironment, necrotic cells released DAMPs into the extracellular space. ${ }^{16}$ The expression of TLRs has been confirmed in various carcinomas, ${ }^{16}$ and those TLRs are thought to be activated in the tumor microenvironment. Regarding their anti-tumorigenic effect, activation of TLRs led to the activation of immune cells, and a consequent induction of apoptosis. Conversely, their pro-tumorigenic effect was expressed as the activation of immunosuppressive cells, with consequent promotion of angiogenesis. ${ }^{16-18}$

In CRC, the expressions of TLR2, TLR3, TLR4, TLR5, and TLR9 has been confirmed. ${ }^{16}$ Nihon-Yanagi et al suggested that TLR2 expression might be involved in sporadic colorectal carcinogenesis. ${ }^{19}$ So et al suggested that TLR4 expression in tumor cells promotes tumor progression. ${ }^{20}$ Eiro et al suggested a possible protective role of TLR9 expression against malignant transformation in CRC. ${ }^{21}$ These research about TLRs on CRC were only in vitro studies, and no clinicopathological study or investigation of the association of TRLs with clinical outcomes have been conducted.

TLR3 recognizes virus-derived double-stranded RNA (dsRNA), synthetic dsRNA, and polyinosinic-polycytidylic acid (poly I:C), and induces the activation of type
I interferons (IFNs), leading to the initiation of an antiviral response. ${ }^{22}$ Also, messenger RNA (mRNA) released from necrotic cancer cells and self dsRNA, acting as DAMPs, activated TLR3 expression. ${ }^{16,23}$ TLR3 has been reported to have antitumor activities and may be a favorable prognostic factor since TLR3 activation is involved in the apoptosis and inhibition of the proliferation of breast tumors, melanomas, prostate cancers, and hepatocellular carcinoma cells. ${ }^{24-27}$ Also, a favorable outcome was found for patients with hepatocellular carcinoma who were TLR3-positive on immunohistochemistry, as opposed to the outcome for TLR3-negative patients. ${ }^{27}$

In this study, we examined TLR3 expression in CRC, and the clinicopathological factors and outcomes to evaluate the significance of TLR3 expression in CRC. Furthermore, cytokines induced by TLR3 activation were investigated in vitro in a cultured human CRC-derived cell line. In addition, we examined whether TLR3 expression in surgical specimens was involved in the secretion of these induced cytokines.

\section{Patients and Methods}

\section{Patients}

We obtained data on the 5-year outcomes in 113 of 264 patients who underwent surgery for CRC at the Hirosaki University School of Medicine Hospital between January 2010 and December 2011. The subjects were 50 patients who were randomly extracted from these 113 patients using a random number table.

\section{Data Collection and Definitions}

Clinical information (sex, age at time of surgery, and date of diagnosis), clinicopathological information (region occupied by tumor, histologic type, histological invasion depth, stromal volume, local advancement pattern, vascular invasion, tumor budding, lymph node metastasis, distant metastasis, and disease stage), and postoperative information (recurrence, date of recurrence, and outcome) were collected. Pathological diagnoses were based on the criteria used in the Japanese Classification of Colorectal Carcinoma, 7th edition. The right colon was defined as the region from the cecum to the transverse colon, the left colon as the region from the descending colon to the rectum, the colon as the region from the cecum to the rectosigmoid. Overall survival (OS) was defined as the period from the diagnosis to final follow-up, and recurrence-free survival (RFS) as the period from the day of curative resection (R0) to recurrence. The 
Table I Primer Sequences

\begin{tabular}{|l|l|l|}
\hline Primer Name & Sequence (5' to $\mathbf{3}^{\prime}$ ) & Bases \\
\hline CCL2 - forward & AAA CTG AAG CTC GCA CTC TCG C & 353 \\
CCL2 - reverse & ATT CTT GGG TTG TTG AGT GAG T \\
CCL5 - forward & CTA CTC GGG AGG CTA AGG CAG GAA & 318 \\
CCL5 - reverse & GAG GGG TTG AGA CGG CGG AAG C & \\
IL-8 - forward & AGG AGT GCT AAA GAA CTT CGA & 219 \\
IL-8 - reverse & TGA ATT CTC AGC CCT CTT CAA & \\
GAPDH - forward & GCA CCG TCA AGG CTG AGA AC & 142 \\
GAPDH - reverse & ATG GTG GTG AAG ACG CCA GT & \\
\hline
\end{tabular}

day of curative resection was defined as the first operation day in stage I-III cases without distant metastasis. In stage IV cases with distant metastasis, the day of curative resection was defined as the day of operation for distant metastasis after surgery for the primary lesion at the first surgery. Curative resection was achieved in 45 of the 50 patients, excluding 5 patients in whom curative resection for distant metastasis was not applicable.

\section{Immunohistochemistry and Assessment}

Immunohistochemistry (IHC) was performed on formalinfixed, paraffin-embedded tissue sections using standard techniques with a BOND-III fully automatic immunostaining device (Leica Camera AG, Wetzlar, Germany). Antigen retrieval was achieved using BOND Epitope Retrieval
Solution 1 (ER1, a citrate based $\mathrm{pH} 6.0$ solution, Leica Camera AG) for interleukin-8 (IL-8), and BOND Epitope Retrieval Solution 2 (ER2, an EDTA-based pH 9.0 solution, Leica Camera AG) for the rest. Following antigen retrieval, tissue samples were incubated for $15 \mathrm{~min}$ with mouse monoclonal antibodies against TLR3 (NBP2-24875, Novus Biologicals, Centennial, $\mathrm{CO}$ ) at 1:30 dilution, for 20 min with rabbit polyclonal antibodies against C-C motif chemokine ligand 2 (CCL2) (HPA019163, Sigma-Aldrich, St. Louis, MO) at 1:50 dilution, 20 min with goat polyclonal antibodies against $\mathrm{C}-\mathrm{C}$ motif chemokine ligand 5 (CCL5) (AF-278-NA, R\&D Systems, Minneapolis, MN) at 1:25 dilution and for $20 \mathrm{~min}$ with rabbit polyclonal antibodies against IL-8 (AHC0881, Thermo Fisher Scientific, Waltham, MA) at 1:100 dilution. BOND Polymer Refine

Table 2 Patient Characteristics

\begin{tabular}{|c|c|c|c|c|c|}
\hline Item & Value & Item & Value & Item & Value \\
\hline Sex: male (\%) & $30(60.0)$ & Tumor invasion (\%) & & Venous invasion (\%) & \\
\hline Age at surgery $(\mathrm{y})$ (median [IQR]) & $66.50[57.50-74.00]$ & pSM & $4(8.0)$ & v0 & $6(12.0)$ \\
\hline Tumor location (\%) & & pMP & II (22.0) & vl & $17(34.0)$ \\
\hline Cecum & $2(4.0)$ & pSS, pA & $26(52.0)$ & v2 & $16(32.0)$ \\
\hline Ascending colon & $10(20.0)$ & pSE & $7(14.0)$ & v3 & II (22.0) \\
\hline Transverse colon & $4(8.0)$ & pSI, pAI & $2(4.0)$ & Tumor budding (\%) & \\
\hline Descending colon & $2(4.0)$ & Stromal vol. (\%) & & I & $31(62.0)$ \\
\hline Sigmoid colon & $9(18.0)$ & med & $5(10.0)$ & 2 & II (22.0) \\
\hline RS & $6(12.0)$ & int & $43(86.0)$ & 3 & $8(16.0)$ \\
\hline $\mathrm{Ra}$ & $4(8.0)$ & sci & $2(4.0)$ & LN metastasis (\%) & \\
\hline $\mathrm{Rb}$ & $13(26.0)$ & INF (\%) & & $\mathrm{pN} 0$ & $20(40.0)$ \\
\hline Differentiation (\%) & & INF a & $3(6.0)$ & $\mathrm{pNI}$ & $14(28.0)$ \\
\hline Pap & I (2.0) & INF b & $37(74.0)$ & $\mathrm{pN} 2$ & $12(24.0)$ \\
\hline Well & $5(10.0)$ & INF c & $10(20.0)$ & $\mathrm{pN} 3$ & $4(8.0)$ \\
\hline Mod & $36(72.0)$ & Lymphatic invasion (\%) & & Metastasis (\%) & \\
\hline Por & $6(12.0)$ & ly0 & $5(10.0)$ & Mo & $40(80.0)$ \\
\hline Sig & I (2.0) & $|y|$ & $21(42.0)$ & MI & $10(20.0)$ \\
\hline Muc & I (2.0) & ly2 & $16(32.0)$ & & \\
\hline & & ly3 & $8(16.0)$ & & \\
\hline
\end{tabular}


Detection and BOND DAB Enhancer (Leica Camera AG) was used for detecting primary antibodies. Staining intensity for TLR3 was evaluated as negative, weakly positive, or strongly positive by a pathologist who was blinded to the clinical information. Immunostainings for CCL2, CCL5 and IL-8 were examined in tissues known to be positive and negative for TLR3 expression.

\section{Reagents}

Poly I:C and anti-actin rabbit $\operatorname{IgG}$ were obtained from Sigma-Aldrich. Non-silencing negative control smallinterfering RNA (siRNA) (1027281) and siRNA against TLR3 (SI02655156) were purchased from Qiagen (Hilden, Germany). Lipofectamine RNAi MAX was purchased from Invitrogen (Carlsbad, CA). A NucleoSpin RNA kit was obtained from Takara-Bio (Shiga, Japan). Oligonucleotide primers for the reverse transcriptionpolymerase chain reaction (RT-PCR) and an oligo(dT) 18 primer were synthesized by Greiner Japan (Tokyo, Japan). dNTP mix and Moloney murine leukemia virus reverse-transcriptase (M-MLV RT) were obtained from Thermo Fisher Scientific. SsoAdvanced Universal SYBR
Green Supermix was purchased from Bio-Rad (Hercules, CA). Precast polyacrylamide gels were purchased from ATTO (Tokyo, Japan). Enzyme-linked immunosorbent assay (ELISA) kits for CCL2, CCL5, and IL-8 were purchased from R\&D Systems.

\section{Cell Culture}

The human CRC cell line SW480 (ATCC, Rockville, MD) was cultured in RPMI 1640 medium containing 10\% fetal bovine serum (FBS) (Thermo Fisher Scientific) combined with amphotericin $\mathrm{B}$, penicillin, and streptomycin under $5 \% \mathrm{CO}_{2}$ at $37^{\circ} \mathrm{C}$.

\section{Poly I:C Treatment and siRNA Transfection}

SW480 was treated with poly I:C at a concentration of 0.08 $50 \mu \mathrm{g} / \mathrm{mL}$ and incubated for $24 \mathrm{~h}$. For transfection with nonsilencing control siRNA or siRNA against TLR3, the medium was changed to one containing no antibiotic from the day before transfection, and cells were treated with siRNA for $4 \mathrm{~h}$ using Lipofectamine RNAi MAX for transfection.

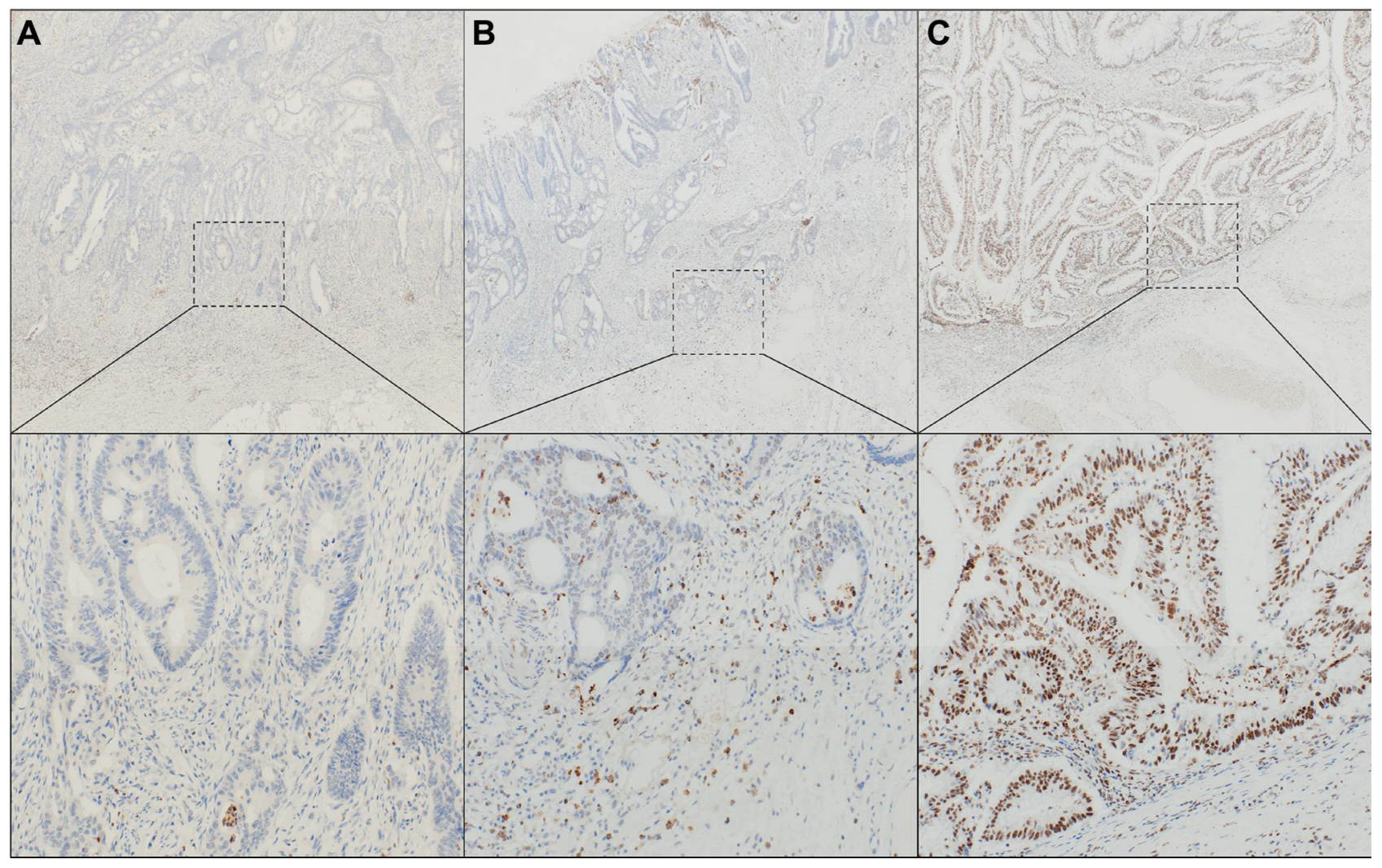

Figure I Immunohistochemistry for TLR3. (A) TLR3 was not stained for tumor cells. (B) TLR3 was weakly stained around the nucleus. (C) TLR3 was strongly stained around the nucleus and weakly for the cytoplasm (Magnification: upper 40×, lower 200x). 
The medium was then replaced with one containing antibiotics, and cells were treated with $30 \mu \mathrm{g} / \mathrm{mL}$ poly I:C for 4 to 24 $\mathrm{h}$ on the following day. The cells were then used for quantitative real-time RT-PCR and Western blot analyses, and the conditioned medium was used for ELISA.

\section{Quantitative Real-Time RT-PCR Analysis}

Total RNA was extracted using a NucleoSpin RNA kit. Single-stranded complementary DNA (cDNA) was synthesized from $1 \mu \mathrm{g}$ of total RNA using M-MLV RT. For amplification of the cDNAs of CCL2, CCL5, IL-8, and glyceraldehyde-3-phosphate dehydrogenase (GAPDH), SsoAdvanced Universal SYBR Green Supermix was used. The primers used for quantitative real-time RT-PCR are shown in Table 1. Each sample was measured in triplicate.

\section{Western Blot Analysis}

After incubation, the cells were dissolved with Laemmli's reducing sample buffer. Lysates were used in $7.5 \%$ polyacrylamide gel electrophoresis, and separated proteins were transferred to polyvinylidene difluoride membranes. These membranes were blocked and then incubated with anti-TLR3 (1:500) and anti-actin (1:5000) antibodies, followed by incubation with horseradish peroxidase-conjugated secondary antibody. Bands were detected using chemiluminescence.

\section{ELISA for Measurement of CCL2, CCL5 and IL-8 Proteins in Culture Medium}

CCL2, CCL5, and IL-8 levels in the conditioned medium were measured using ELISA kits.

Table 3 Clinicopathological Factors in TLR3-Negative and TLR3-Positive Cases on IHC

\begin{tabular}{|c|c|c|c|}
\hline Variables & TLR3-Negative [N=14] & TLR3-positive $[\mathrm{N}=36]$ & $p$-value \\
\hline Sex: male (\%) & $10(71.4)$ & $20(55.6)$ & 0.35 \\
\hline Age at surgery $(y)$ : median [IQR] & $64.00[54.50-73.75]$ & $66.50[59.75-74.00]$ & 0.64 \\
\hline \multicolumn{4}{|l|}{ Tumor location (\%) } \\
\hline Right/Left & $7(50.0) / 7(50.0)$ & $9(25.0) / 27(75.0)$ & 0.11 \\
\hline Colon/Rectum & $9(64.3) / 5(35.7)$ & $24(66.7) / 12(33.3)$ & 1 \\
\hline \multicolumn{4}{|l|}{ Differentiation (\%) } \\
\hline Well-mod/por & $12(85.7) / 2(14.3)$ & $30(83.3) / 6(16.7)$ & I \\
\hline \multicolumn{4}{|l|}{ Tumor invasion (\%) } \\
\hline PTI-2/pT3- & $3(21.4) / 11$ (78.6) & $12(33.3) / 24(66.7)$ & 0.51 \\
\hline \multicolumn{4}{|l|}{ Stromal vol. (\%) } \\
\hline Med/int/sci & I $(7.1) / 13(92.9) / 0$ & $4($ II.I)/30 (83.3)/2 (5.6) & I \\
\hline \multicolumn{4}{|l|}{ INF (\%) } \\
\hline INF a/b/c & I (7.I)/II (78.6)/2 (14.3) & $2(5.6) / 26(72.2) / 8(22.2)$ & 0.87 \\
\hline Lymphatic invasion: Ly+ (\%) & $12(85.7)$ & $33(91.7)$ & 0.61 \\
\hline Venous invasion: $\mathrm{V}+(\%)$ & $13(92.9)$ & $31(86.1)$ & 0.66 \\
\hline Tumor budding: high (2 or 3 ) (\%) & $8(57.1)$ & II (30.6) & 0.11 \\
\hline LN metastasis: pN+ (\%) & $12(85.7)$ & $18(50.0)$ & 0.03 \\
\hline Metastasis: MI (\%) & $3(21.4)$ & $7(19.4)$ & 1 \\
\hline \multicolumn{4}{|l|}{ pStage (\%) } \\
\hline I & I (7.I) & $9(25.0)$ & 0.12 \\
\hline II & I (7.I) & $9(25.0)$ & \\
\hline Illa & $6(42.9)$ & $4(11.1)$ & \\
\hline IIllb & $3(21.4)$ & $7(19.4)$ & \\
\hline IV & $3(21.4)$ & $7(19.4)$ & \\
\hline Outcome: alive (\%) & $10(7 \mid .4)$ & $28(77.8)$ & 0.72 \\
\hline
\end{tabular}

Note: Categorical variables by Fisher exact test, continuous variables by Mann-Whitney U-test. 


\section{Statistical Analyses}

To evaluate patients' background, Fisher's exact test was used to analyze nominal variables, and a Mann-Whitney $U$-test was used for continuous variables. In the univariate analysis, OS and RFS were calculated using the Kaplan-Meier method and analyzed using the Log rank test. In the multivariate analysis of RFS, a Cox proportional hazards model was employed. Quantitative real-time PCR and ELISA data are shown as the mean $\pm \mathrm{SD}(\mathrm{n}=3)$, with a Student's $t$-test used for analysis. $P$ $<0.05$ was regarded as significant in each analysis. All statistical analyses were performed using EZR (Saitama Medical Center, Jichi Medical University, Japan), a graphical user interface for R (The R Foundation for Statistical Computing, Vienna, Austria). ${ }^{28}$

\section{Results}

\section{Patient Characteristics}

The patient characteristics of the 50 patients ( $60 \%$ male) with CRC is shown in Table 2. The median age at the time of surgery was 66.5 years old. The median (IQR) duration of follow-up was 64.0 months (range, 51.4-76.3 months). Lesions were located in the colon in 33 patients $(66 \%)$.
The histologic type was well-differentiated in 42 patients (84\%). The depth of tumor invasion was submucosa (SM) in 4 patients, and the invasion distance exceeded $1000 \mu \mathrm{m}$ in these patients. Distant metastasis was present in 10 patients $(20 \%)$ as at the first surgery, and chemotherapy was performed after surgery for the primary lesion, followed by surgery for distant metastasis in 5 of these cases.

\section{TLR3 Expression in CRC Correlates with Lymph Node Metastasis}

Of the 50 patients, $14(28 \%)$ were TLR3-negative on IHC (Figure 1A), 21 (42\%) were weakly positive, and 15 $(30 \%)$ were strongly positive (Figure $1 \mathrm{~B}$ and C). The stained region was located mainly around the nucleus. Perinuclear stainability was strong in strongly positive TLR3 cases, and staining was also noted in the cytoplasm in these cases. Weakly and strongly positive cases were combined as a TLR3-positive group for comparison with

TLR3-negative cases (Table 3). These groups showed no significant differences in sex, age, tumor location, invasion depth, stromal volume, local advancement pattern, vascular invasion, tumor budding, or distant metastasis,

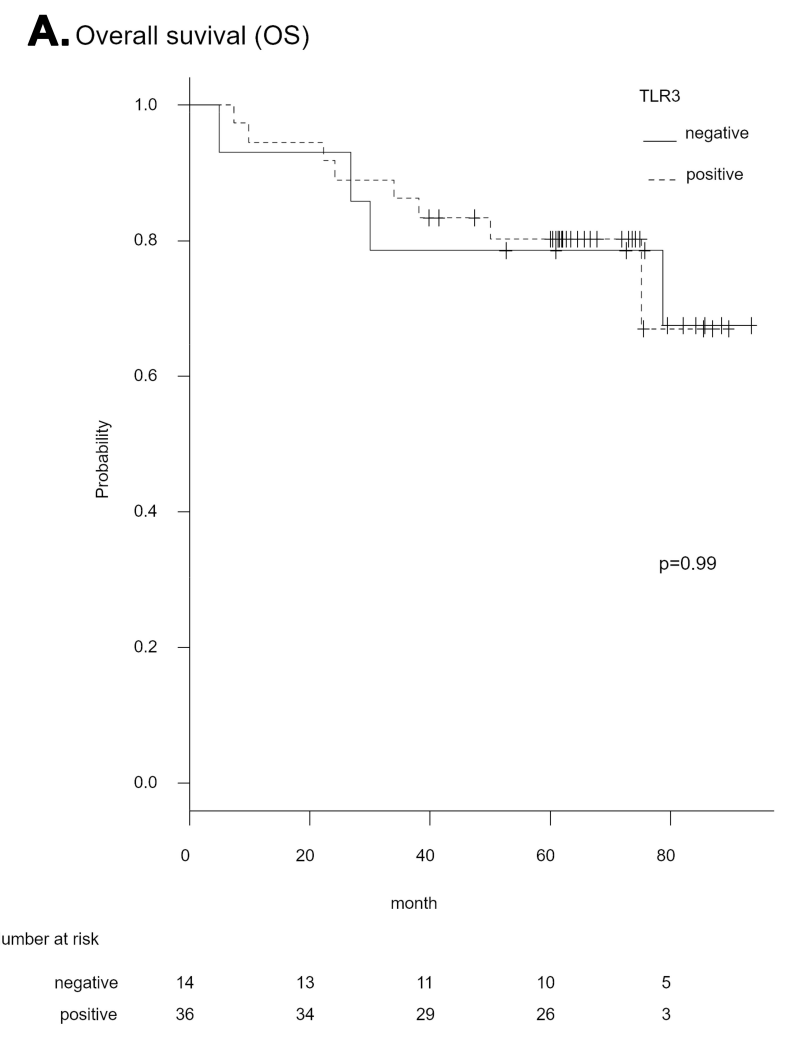

B. Recurrence-free survival (RFS)

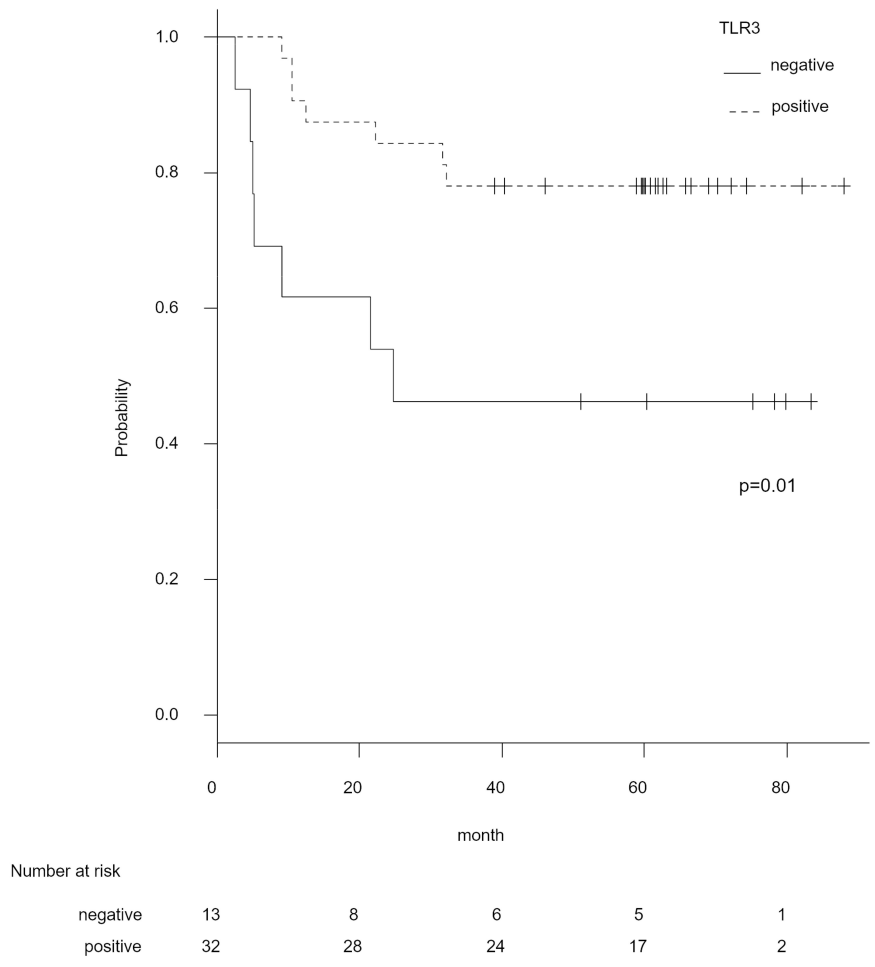

Figure 2 The association of TLR3 with OS and RFS in CRC. We compared OS (A) and RFS (B) in both TLR3-positive and -negative groups. (A) There was no significant difference in OS in both groups (TLR3-positive: $80.2 \%$ vs TLR3-negative: 78.6\%). (B) On the other hand, the TLR3-negative group had a significantly lower RFS than did the TLR3-positive group (TLR3-positive: 78.1\% vs TLR3-negative: $46.2 \%$ ), indicating that the TLR3-positive group had significantly less recurrence. 
but there were more lymph node metastasis-positive cases in the TLR3-negative group (85.7\%) than TLR3-positive group $(50.0 \%)$, and this difference was statistically significant $(p=0.03)$.

\section{TLR3-Negative CRC is a Risk Factor for Recurrence}

OS and RFS in the TLR3-negative and -positive groups are shown in Figure 2. There was no difference in OS between the two groups, but the 5-year RFS rate of $46.2 \%$ in the TLR3-negative group was significantly lower than that of $78.1 \%$ in the TLR3-positive group $(p=0.01)$. The results of the univariate analysis of 5-year RFS with differentiation grade, invasion depth, vascular invasion, tumor budding, and lymph node metastasis, and TLR3 (all factors thought to be related to recurrence) are shown in Table 4. Recurrence tended to occur in advanced stage cases, but there was no significant difference for differentiation grade, invasion depth, or vascular invasion. However, the 5-year RFS was significantly shorter in patients with a high tumor budding, lymph node metastasis, and a TLR3-negative status. In the multivariate analysis using a Cox proportional hazards model with these three factors, a high tumor budding (hazard ratio [HR]: 7.52, 95\% confidence interval [CI]: 2.07-27.33, $p$ $<0.01$ ) and a TLR3-negative status (HR: 3.05, 95\% CI: $1.05-8.83, p=0.04$ ) were identified as independent risk factors for recurrence (Table 5).

\section{TLR3 is Expressed in SW480 Cells, and Poly I:C Induces CCL2, CCL5, and IL-8 in These Cells}

The expression of TLR3 was examined by Western blot analysis after treating SW480 cells with $0-50 \mu \mathrm{g} / \mathrm{mL}$ poly I:C for $24 \mathrm{~h}$ (Figure 3). TLR3 was expressed low levels and steady state in these cells, and there was no poly I:Cinduced change. Upon treatment of the cells with poly I:C, mRNA levels for CCL2, CCL5, and IL-8 increased, peaked within 4 hours, and then decreased (Figure 4A-C). Poly I:C also induced mRNA and protein expression of these chemokines in a concentration-dependent manner (Figure 4D-I).

Table 4 Univariate Analysis of 5-Year Recurrence-Free Survival (RFS)

\begin{tabular}{|c|c|c|c|}
\hline Univariate Analysis & $\mathbf{N}$ & 5-Year RFS (95\% CI) & $p$-value \\
\hline \multicolumn{4}{|l|}{ Differentiation } \\
\hline High (pap, tub) & 38 & $0.737(0.566-0.849)$ & 0.18 \\
\hline Low (por, sig, muc) & 7 & $0.429(0.098-0.734)$ & \\
\hline \multicolumn{4}{|l|}{ Tumor invasion } \\
\hline PTI-2 & 15 & $0.867(0.564-0.965)$ & 0.07 \\
\hline pT3-4 & 30 & $0.600(0.405-0.750)$ & \\
\hline \multicolumn{4}{|l|}{ Lymphatic invasion } \\
\hline Ly0 & 5 & $0.800(0.204-0.969)$ & 0.65 \\
\hline Ly+ & 40 & $0.675(0.507-0.797)$ & \\
\hline \multicolumn{4}{|l|}{ Venous invasion } \\
\hline Vo & 6 & $0.833(0.273-0.975)$ & 0.48 \\
\hline $\mathrm{V}+$ & 39 & $0.667(0.496-0.791)$ & \\
\hline \multicolumn{4}{|l|}{ Tumor budding } \\
\hline Low (I) & 28 & $0.893(0.704-0.964)$ & $<0.01$ \\
\hline High (2-3) & 17 & $0.353(0.145-0.570)$ & \\
\hline \multicolumn{4}{|l|}{ Lymph node metastasis } \\
\hline $\mathrm{pN} 0$ & 20 & $0.900(0.656-0.974)$ & $<0.01$ \\
\hline $\mathrm{pN+}$ & 25 & $0.520(0.312-0.692)$ & \\
\hline \multicolumn{4}{|l|}{ TLR3 } \\
\hline Negative & 13 & $0.462(0.192-0.696)$ & 0.01 \\
\hline Positive & 32 & $0.78 \mid(0.595-0.889)$ & \\
\hline
\end{tabular}

Note: Log rank test. 
Table 5 Results of Multivariate Analysis of Factors with a Potential Association with 5-Year Recurrence-Free Survival Using a Cox Proportional Hazard Model

\begin{tabular}{|l|l|l|}
\hline Multivariate Analysis & Hazard Ratio $(\mathbf{9 5} \%-\mathbf{C l})$ & $\mathbf{p}$-value \\
\hline Tumor budding: high & $7.52(2.07-27.33)$ & $<0.01$ \\
LN metastasis: $\mathrm{pN+}$ & $2.52(0.48-13.12)$ & 0.27 \\
TLR3: negative & $3.05(1.05-8.83)$ & 0.04 \\
\hline
\end{tabular}

\section{TLR3 is Involved in Poly I:C-Induced CCL2, CCL5, and IL-8 Expression}

The introduction of siRNA against TLR3 inhibited poly I: C-induced expression of CCL2, CCL5, and IL-8 mRNA and protein (Figure $5 \mathrm{~A}-\mathrm{F}$ ), showing that these processes are dependent on TLR3.

\section{TLR3-Positive CRC Surgical Specimens Demonstrated Expression of CCL2, CCL5 and IL-8}

Immunostainings for CCL2, CCL5 and IL-8 of TLR3-positive CRC surgical specimens are shown in Figure 6. CCL2 and IL-8 stained weakly in the cytoplasm of the tumor cells. In comparison, CCL5 stained uniformly and strongly in the cytoplasm. CCL5 and IL-8 also stained in stromal cells.

\section{Discussion}

The lymph node metastasis-positive rate was high, and the 5-year RFS was short in patients with a TLR3-negative surgical specimen, compared to those who were TLR3positive. Furthermore, TLR3-negative expression was an independent risk factor for recurrence. In cultured cells, TLR3 activation promoted the induction of CCL2, CCL5, and IL-8 in a TLR3-positive CRC-derived cell line (SW480) indicating dependence on the TLR3 signal. In surgical specimens, the expressions of CCL2, CCL5 and IL-8 were found in the cytoplasm of TLR3-positive CRC. The expressions of CCL5 and IL-8 were also found in stromal cells. This suggests that TLR3 may inhibit CRC growth and metastasis via these chemokine's pathways and may be useful as a new treatment target.

While TLR expression in cancer has been reported to have both pro-tumorigenic and anti-tumorigenic effects, ${ }^{16-18}$ TLR3 has been found to inhibit the growth of various carcinomas. ${ }^{24-27}$ Nojiri et al found that poly I:C transfection of SW480 cells activated TLR3 and induced apoptosis in CRC, ${ }^{29}$ whereas Niedzielska et al showed that TLR3 mRNA expression tended to decrease in adenocarcinoma than in a polyp. ${ }^{30}$ In the present study, positive TLR3 expression also
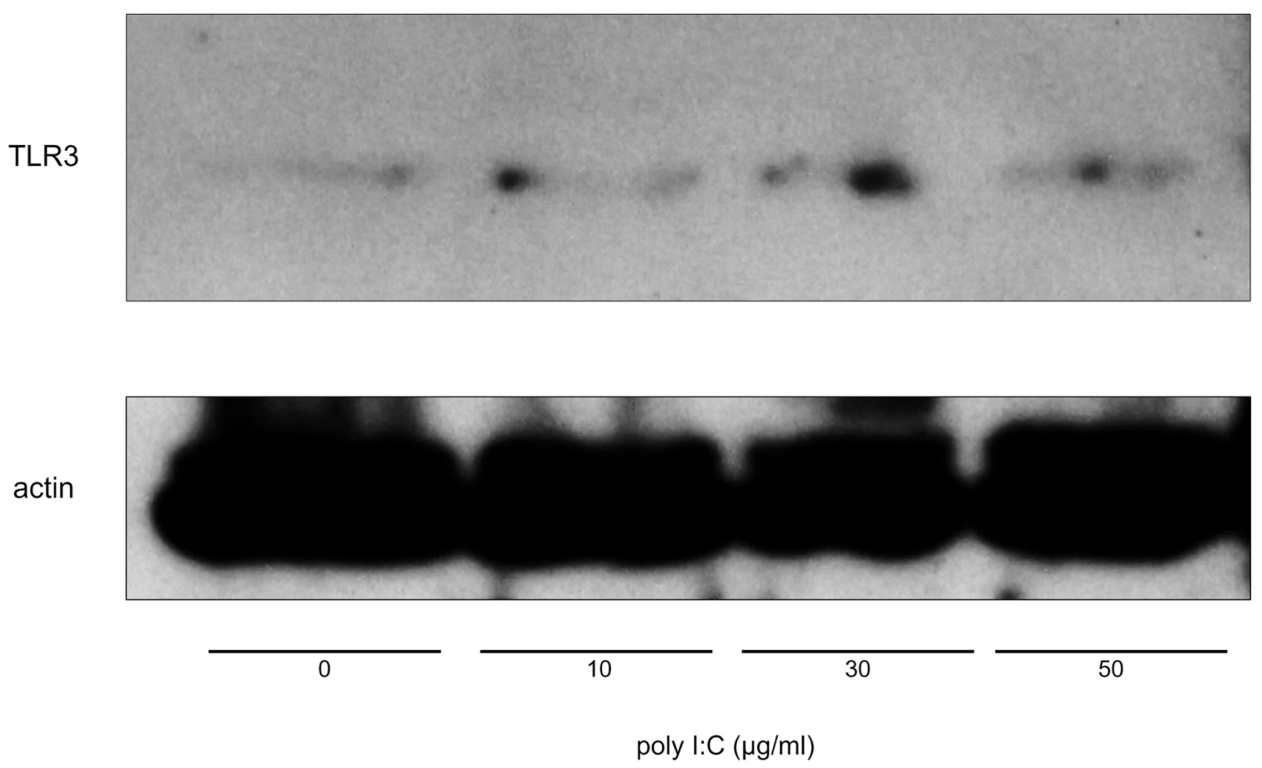

Figure 3 Western blot analysis. TLR3 is expressed in SW480; Cultured SW480 cells were treated with 0-50 $\mu \mathrm{g} / \mathrm{mL}$ poly I:C for 24h and the cells were lysed. The lysate was subjected to Western blot analysis for TLR3 and actin. Expression of a small amount of TLR3 protein was detected even in cells without treatment with poly I:C. Significant upregulation of TLR3 protein by poly I:C was not observed. 
A

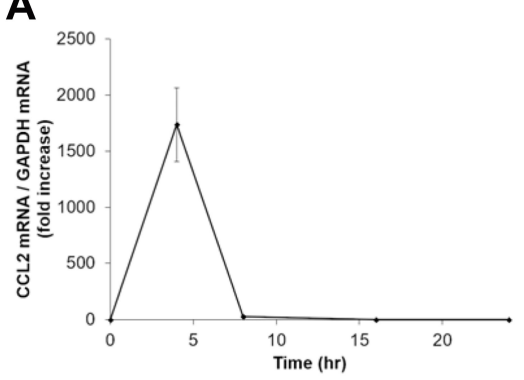

D

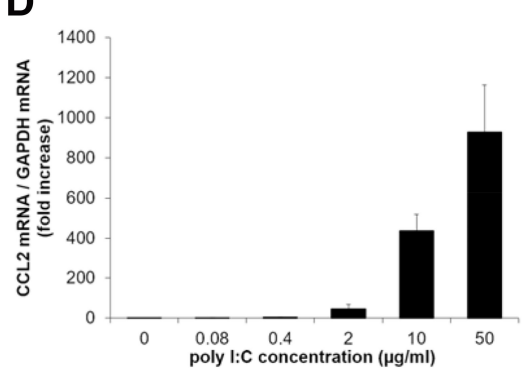

G

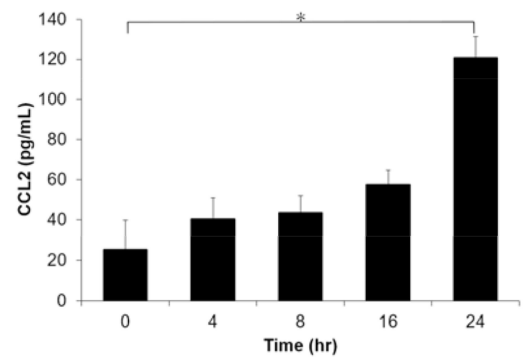

B

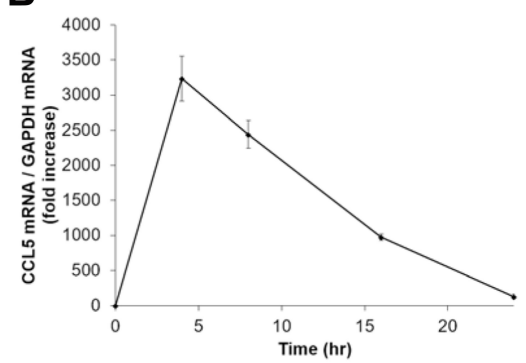

E

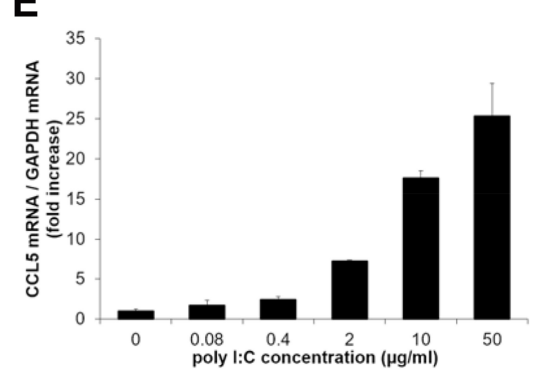

H

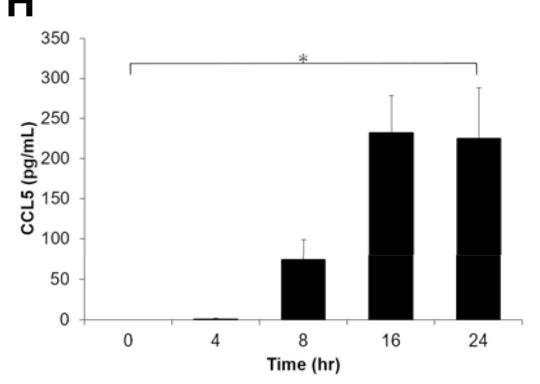

C

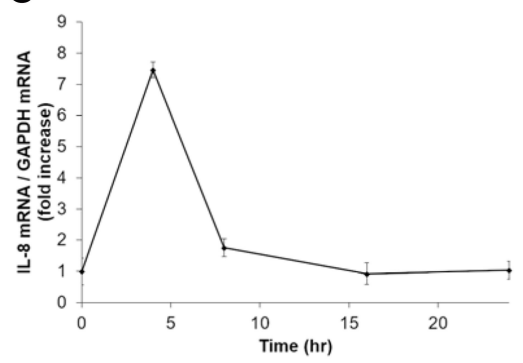

F

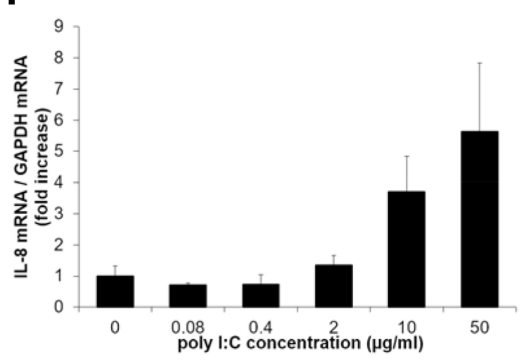

I

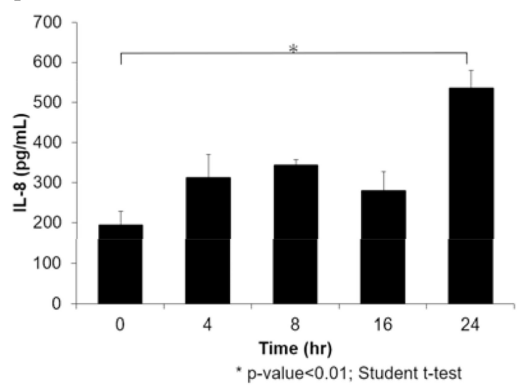

Figure 4 Treatment of SW480 with poly I:C induces the expression of CCL2, CCL5, and IL-8. (A-C) SW480 was treated with $30 \mu \mathrm{g} / \mathrm{mL}$ poly I:C for up to $24 \mathrm{~h}$, and RNA was extracted. The expression of CCL2, CCL5, and IL-8 mRNA was examined using quantitative real-time RT-PCR analysis. Poly I:C induced these mRNA in a time-dependent manner, and the expression peaked at $4 \mathrm{~h}$. (D-F) The cells were treated with $0-50 \mu \mathrm{g} / \mathrm{mL}$ poly I:C for $4 \mathrm{~h}$ and quantitative real-time RT-PCR was performed. Treatment of SW480 with poly I:C induced the expression of these mRNA in a concentration-dependent manner. (G-I) The cells were treated with poly $\mathbf{I}: \mathbf{C}$ as in (A-C), and the medium was collected. The concentration of CCL2, CCL5, and IL-8 proteins was measured using ELISAs. Poly I:C induced expression of CCL2, CCL5, and IL-8 proteins in SW480. *p-value < 0.01 ; Student's t-test.

reduced the lymph node metastasis rate and the recurrence rate, supporting the results of previous reports on various carcinomas. Furthermore, this is the first report on the clinicopathological association of TLR3 with outcomes in CRC.

Many studies on the relationship between CRC and chemokines have been reported. ${ }^{31}$ Generally, CCL2 induces macrophages in inflammatory regions and induces tumorassociated macrophages around tumors. ${ }^{32}$ Watanabe et al found that a reduced CCL2 level in CRC cells is related to lymph node metastasis on immunostaining and a poor outcome, ${ }^{33}$ suggesting that CCL2 is tumor-suppressive. Musha et al reported that CCL5 induced CD8+ T cells in $\mathrm{CRC}$, and this was related to a favorable outcome. ${ }^{34}$ Daster et al suggested that a high frequency of CD8 $+\mathrm{T}$ cell infiltration is significantly predictive of a lack of lymph node metastasis in early rectal cancer. ${ }^{35}$ According to Kobayashi,
IL-8 has a highly neutrophil-selective chemotactic activity. ${ }^{36}$ In addition, Granot et al showed that tumor-entrained neutrophils had cytotoxic activity, killed tumor cells, and inhibited metastasis. ${ }^{37}$ In the present study, TLR3 expression in CRC patients was involved in the reduction of lymph node metastasis and recurrence rates. Furthermore, CCL2, CCL5, and IL-8 expressions were induced in a TLR3-dependent manner in TLR3-positive CRC cells, and these chemokines' expression was observed in TLR3-positive CRC in surgical specimens. These results suggest that the TLR3-mediated induction of the secretion of these chemokines in CRC may have inhibited lymph node metastasis through stromal cells such as macrophages, $T$ cells, and neutrophils.

One limitation of this study is that the direct effects of these chemokines on tumor cells or stromal cells were not investigated. Similar to TLR, chemokines have been 
A

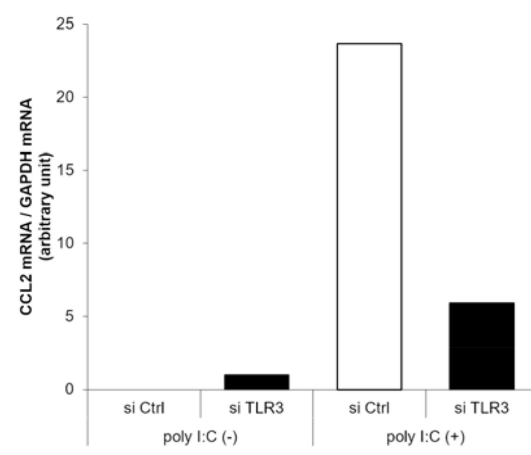

D

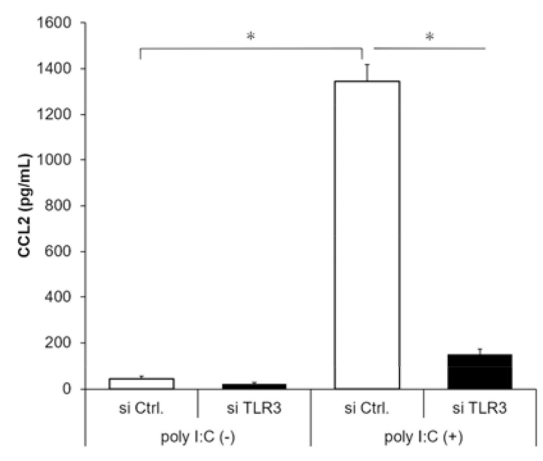

B

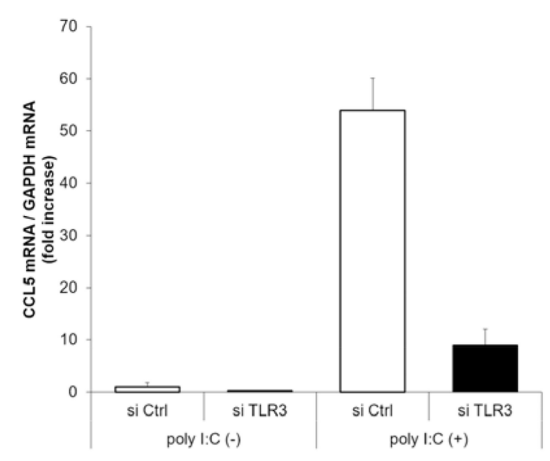

E

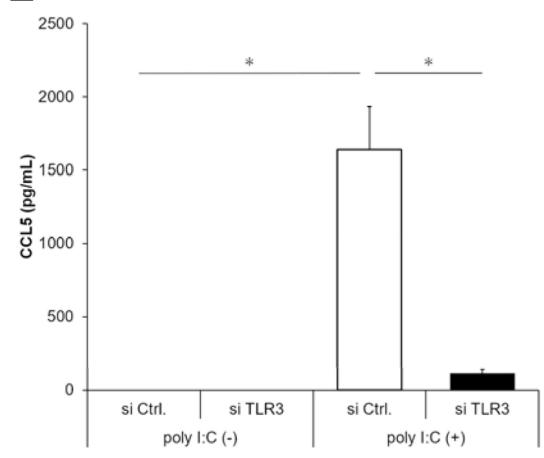

C

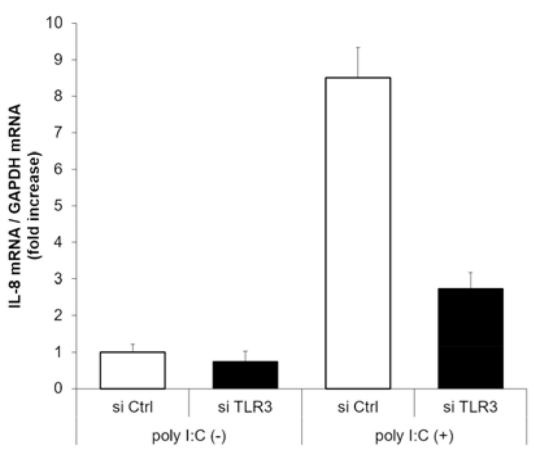

F

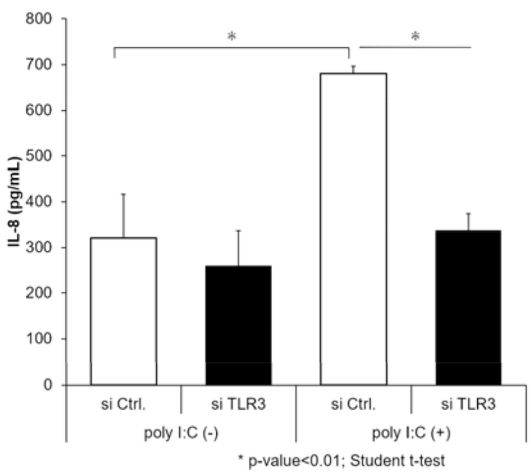

Figure 5 TLR3 is involved in the expression CCL2, CCL5, and IL-8 induced by poly I:C. SW480 were transfected with siRNA against TLR3 or non-silencing control siRNA. After $48 \mathrm{~h}$, the cells were treated with poly I:C. After further incubation for $4 \mathrm{~h}$ and $24 \mathrm{~h}$, RNA was extracted, and the conditioned medium was collected. The expressions of mRNA (A-C) and protein (D-F) for CCL2, CCL5, and IL-8 were estimated using quantitative real-time RT-PCR and ELISA, respectively. Expression of all of CCL2, CCL5, and IL-8 was upregulated by poly I:C and siRNA against TLR3 inhibited the upregulation of these molecules. *P-value < 0.0 I; Student's $t$-test.

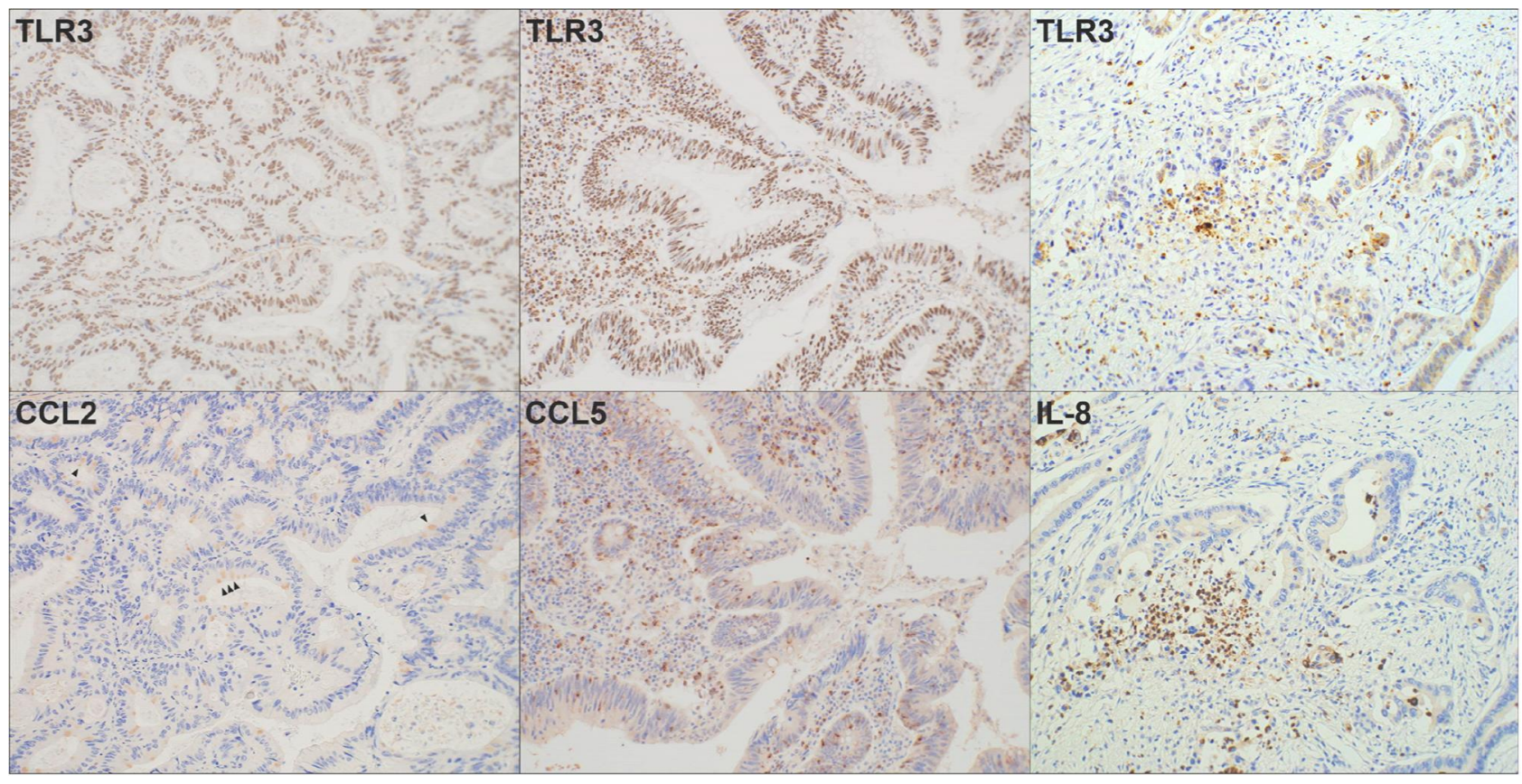

Figure 6 Immunohistochemistry for CCL2, CCL5 and IL-8 in TLR3-positive CRC in surgical specimens. In TLR3-positive CRC specimens, CCL2 was partially stained in the cytoplasm of tumor cells (arrow head). CCL5 was uniformly stained in the cytoplasm of the tumor cells and was also found in the surrounding stromal cells. IL-8 was uniformly and faintly stained in the cytoplasm of tumor cells and also stained in stromal cells (Magnification: 200x). 
reported to have both pro-tumorigenic and anti-tumorigenic effects in the tumor microenvironment. ${ }^{38}$ To further evaluate whether secreted chemokines can suppress metastasis through stromal cells, it would be necessary to coculture CRC cells and stromal cells to examine the interactions that occur when TLR3 is activated.

The present study indicated that the non-expression of TLR3 in CRC cells was associated with lymph node metastasis, and was an independent risk factor for recurrence. Risk factors for recurrence of CRC are mostly surgical and pathological. However, further studies on the association between CRC and TLR3 may lead to the development of new therapeutic strategies, using TLR3 as a molecular target and a prognosticator for recurrence in CRC.

\section{Conclusion}

In summary, the non-expression of TLR3 in CRC cells was associated with lymph node metastasis and was an independent risk factor for recurrence. This is the first report showing the relationship between TLR3 and prognosis in CRC. These results suggest that TLR3 may not only be used as a risk factor for recurrence and a prognostic factor, but further studies on the involvement of TLR3 with tumor growth may provide new molecular targeting therapeutic strategies.

\section{Abbreviations}

CRC, colorectal cancer; TLRs, toll-like receptors; PAMPs, pathogen-associated molecular patterns; DAMPs, damageassociated molecular patterns; HMGB1, high mobility group 1; dsRNA, double-stranded RNA; poly I:C, polyinosinic-polycytidylic acid; IFNs, interferons; mRNA, messenger RNA; OS, overall survival; RFS, recurrencefree survival; IHC, immunohistochemistry; siRNA, smallinterfering RNA; PCR, polymerase chain reaction; ELISA, enzyme-linked immunosorbent assay; CCL2, C-C motif chemokine ligand 2; CCL5, C-C motif chemokine ligand 5; IL-8, interleukin-8; GAPDH, glyceraldehyde-3-phosphate dehydrogenase.

\section{Data Sharing Statement}

The datasets analyzed in the study are available from the corresponding author on reasonable request.

\section{Ethics Approval and Informed Consent}

This study was a retrospective study and informed consent was obtained from all patients in an opt-out format. This study was approved by the ethics committee of Hirosaki University Graduate School of Medicine (reference number: 2018-100). All procedures performed in studies involving human participants were per the ethical standards of the institutional and national committee and the 1964 Helsinki Declaration and its later amendments or comparable ethical standards.

\section{Acknowledgments}

We are grateful to Dr. Mitsuru Chiba at Hirosaki University Graduate School of Health Sciences for providing us with the SW480 cells used in the study. We are also grateful to Mao Kawamura and Naoya Kumagai at the Department of Pathology, Hirosaki University Hospital, for their assistance in this study. We would like to thank Editage (www.editage.com) for English language editing.

\section{Author Contributions}

TY, TM and $\mathrm{KH}$ conceived and designed the research project. All authors contributed to data analysis, drafting or revising the article, have agreed on the journal to which the article will be submitted, gave final approval of the version to be published, and agree to be accountable for all aspects of the work.

\section{Funding}

No funding was provided for this study.

\section{Disclosure}

The authors declare that they have no competing interests for this work.

\section{References}

1. Bray F, Ferlay J, Soerjomataram I, Siegel RL, Torre LA, Jemal A. Global cancer statistics 2018: GLOBOCAN estimates of incidence and mortality worldwide for 36 cancers in 185 countries. CA Cancer $J$ Clin. 2018;68(6):394-424. doi:10.3322/caac.21492

2. Kuipers EJ, Grady WM, Lieberman D, et al. Colorectal cancer. Nat Rev Dis Primers. 2015;1:15065. doi:10.1038/nrdp.2015.65

3. Terzic J, Grivennikov S, Karin E, Karin M. Inflammation and colon cancer. Gastroenterology. 2010;138(6):2101-2114.e2105. doi:10.10 53/j.gastro.2010.01.058

4. Tandon K, Imam M, Ismail BE, Castro F. Body mass index and colon cancer screening: the road ahead. World J Gastroenterol. 2015;21 (5):1371-1376. doi:10.3748/wjg.v21.i5.1371

5. Fedirko V, Tramacere I, Bagnardi V, et al. Alcohol drinking and colorectal cancer risk: an overall and dose-response meta-analysis of published studies. Ann Oncol. 2011;22(9):1958-1972. doi:10. 1093/annonc/mdq653

6. Song M, Garrett WS, Chan AT. Nutrients, foods, and colorectal cancer prevention. Gastroenterology. 2015;148(6):1244-1260 e1216. doi:10. 1053/j.gastro.2014.12.035 
7. Watanabe T, Muro K, Ajioka Y, et al. Japanese society for cancer of the colon and rectum (JSCCR) guidelines 2016 for the treatment of colorectal cancer. Int J Clin Oncol. 2018;23(1):1-34. doi:10.1007/ s10147-017-1101-6

8. Benson AB 3rd, Schrag D, Somerfield MR, et al. American Society of Clinical Oncology recommendations on adjuvant chemotherapy for stage II colon cancer. J Clin Oncol. 2004;22(16):3408-3419. doi:10.1200/JCO.2004.05.063

9. Schmoll HJ, Van Cutsem E, Stein A, et al. ESMO Consensus Guidelines for management of patients with colon and rectal cancer. a personalized approach to clinical decision making. Ann Oncol. 2012;23(10):2479-2516. doi:10.1093/annonc/mds236

10. Takeda K, Akira S. Toll-like receptors. Curr Protoc Immunol. 2015;109(1):1412 11-10. doi:10.1002/0471142735.im1412s109

11. Akira S, Uematsu S, Takeuchi O. Pathogen recognition and innate immunity. Cell. 2006;124(4):783-801. doi:10.1016/j.cell.2006.02.015

12. Matzinger P. Tolerance, danger, and the extended family. Annu Rev Immunol. 1994;12:991-1045. doi:10.1146/annurev.iy.12.040194. 005015

13. Scaffidi P, Misteli T, Bianchi ME. Release of chromatin protein HMGB1 by necrotic cells triggers inflammation. Nature. 2002;418 (6894):191-195. doi:10.1038/nature00858

14. Shi Y, Evans JE, Rock KL. Molecular identification of a danger signal that alerts the immune system to dying cells. Nature. 2003;425(6957):516-521. doi:10.1038/nature01991

15. Venereau E, Ceriotti C, Bianchi ME. DAMPs from cell death to new life. Front Immunol. 2015;6(422):422. doi:10.3389/fimmu.2015. 00422

16. Sato Y, Goto Y, Narita N, Hoon DS. Cancer cells expressing toll-like receptors and the tumor microenvironment. Cancer Microenviron. 2009;2(Suppl 1):205-214. doi:10.1007/s12307-009-0022-y

17. Dajon M, Iribarren K, Cremer I. Toll-like receptor stimulation in cancer: A pro- and anti-tumor double-edged sword. Immunobiology. 2017;222(1):89-100. doi:10.1016/j.imbio.2016.06.009

18. Huang B, Zhao J, Unkeless JC, Feng ZH, Xiong H. TLR signaling by tumor and immune cells: a double-edged sword. Oncogene. 2008;27 (2):218-224. doi:10.1038/sj.onc.1210904

19. Nihon-Yanagi Y, Terai K, Murano T, Matsumoto T, Okazumi S. Tissue expression of Toll-like receptors 2 and 4 in sporadic human colorectal cancer. Cancer Immunol Immunother. 2012;61(1):71-77. doi:10.1007/s00262-011-1085-4

20. So EY, Ouchi T. The application of Toll like receptors for cancer therapy. Int J Biol Sci. 2010;6(7):675-681. doi:10.7150/ijbs.6.675

21. Eiro N, Gonzalez L, Gonzalez LO, et al. Study of the expression of toll-like receptors in different histological types of colorectal polyps and their relationship with colorectal cancer. $J$ Clin Immunol. 2012;32(4):848-854. doi:10.1007/s10875-012-9666-3

22. Alexopoulou L, Holt AC, Medzhitov R, Flavell RA. Recognition of double-stranded RNA and activation of NF-kappaB by Toll-like receptor 3. Nature. 2001;413(6857):732-738. doi:10.1038/35099560

23. Kariko K, Ni H, Capodici J, Lamphier M, Weissman D. mRNA is an endogenous ligand for Toll-like receptor 3. J Biol Chem. 2004;279 (13):12542-12550. doi:10.1074/jbc.M310175200

Clinical and Experimental Gastroenterology

\section{Publish your work in this journal}

Clinical and Experimental Gastroenterology is an international, peerreviewed, open access, online journal publishing original research, reports, editorials, reviews and commentaries on all aspects of gastroenterology in the clinic and laboratory. This journal is indexed on American Chemical Society's Chemical Abstracts Service (CAS).
24. Salaun B, Coste I, Rissoan MC, Lebecque SJ, Renno T. TLR3 can directly trigger apoptosis in human cancer cells. J Immunol. 2006;176 (8):4894-4901. doi:10.4049/jimmunol.176.8.4894

25. Salaun B, Lebecque S, Matikainen S, Rimoldi D, Romero P. Toll-like receptor 3 expressed by melanoma cells as a target for therapy? Clin Cancer Res. 2007;13(15 Pt 1):4565-4574. doi:10.1158/1078-0432. CCR-07-0274

26. Paone A, Starace D, Galli R, et al. Toll-like receptor 3 triggers apoptosis of human prostate cancer cells through a PKC-alpha-dependent mechanism. Carcinogenesis. 2008;29(7):1334-1342. doi:10. 1093/carcin/bgn149

27. Chew V, Tow C, Huang C, et al. Toll-like receptor 3 expressing tumor parenchyma and infiltrating natural killer cells in hepatocellular carcinoma patients. J Natl Cancer Inst. 2012;104(23):1796-1807. doi:10.1093/jnci/djs436

28. Kanda Y. Investigation of the freely available easy-to-use software 'EZR' for medical statistics. Bone Marrow Transplant. 2013;48 (3):452-458. doi:10.1038/bmt.2012.244

29. Nojiri K, Sugimoto K, Shiraki K, et al. The expression and function of Toll-like receptors 3 and 9 in human colon carcinoma. Oncol Rep. 2013;29(5):1737-1743. doi:10.3892/or.2013.2322

30. Niedzielska I, Niedzielski Z, Tkacz M, et al. Toll-like receptors and the tendency of normal mucous membrane to transform to polyp or colorectal cancer. J Physiol Pharmacol. 2009;60(Suppl 1):65-71.

31. Itatani $\mathrm{Y}$, Kawada $\mathrm{K}$, Inamoto $\mathrm{S}$, et al. The role of chemokines in promoting colorectal cancer invasion/metastasis. Int $J$ Mol Sci. 2016;17(5):643. doi:10.3390/ijms17050643

32. Sato K, Kuratsu J, Takeshima H, Yoshimura T, Ushio Y. Expression of monocyte chemoattractant protein-1 in meningioma. $J$ Neurosurg. 1995;82(5):874-878. doi:10.3171/jns.1995.82.5.0874

33. Watanabe H, Miki C, Okugawa Y, Toiyama Y, Inoue Y, Kusunoki M. Decreased expression of monocyte chemoattractant protein-1 predicts poor prognosis following curative resection of colorectal cancer. Dis Colon Rectum. 2008;51(12):1800-1805. doi:10.1007/s10350-0089380-7

34. Musha H, Ohtani H, Mizoi T, et al. Selective infiltration of CCR5(+) CXCR3(+) T lymphocytes in human colorectal carcinoma. Int $J$ Cancer. 2005;116(6):949-956. doi:10.1002/ijc.21135

35. Daster S, Eppenberger-Castori S, Hirt C, et al. High frequency of CD8 positive lymphocyte infiltration correlates with lack of lymph node involvement in early rectal cancer. Dis Markers. 2014;2014:792183. doi:10.1155/2014/792183

36. Kobayashi Y. The role of chemokines in neutrophil biology. Front Biosci. 2008;13:2400-2407. doi:10.2741/2853

37. Granot Z, Henke E, Comen EA, King TA, Norton L, Benezra R. Tumor entrained neutrophils inhibit seeding in the premetastatic lung. Cancer Cell. 2011;20(3):300-314. doi:10.1016/j.ccr.2011.08.012

38. Nagarsheth N, Wicha MS, Zou W. Chemokines in the cancer microenvironment and their relevance in cancer immunotherapy. Nat Rev Immunol. 2017;17(9):559-572.
The manuscript management system is completely online and includes a very quick and fair peer-review system, which is all easy to use. Visit http://www.dovepress.com/testimonials.php to read real quotes from published authors. 